\title{
Questionnaire Survey-Based Quantitative Assessment of the Impact of Transitional Care on Self-Management of Patients with Acute Exacerbation of Chronic Obstructive Pulmonary Disease
}

\author{
Wenjie Xu $\mathbb{D}^{1},{ }^{1}$ Hui Hu $\mathbb{D},{ }^{1}$ and Yanjun Mao $\mathbb{D}^{2}$ \\ ${ }^{1}$ Department of Respiratory and Critical Care Medicine No. 1, Shanghai Pulmonary Hospital, China \\ ${ }^{2}$ Nursing Department, Shanghai Pulmonary Hospital, China \\ Correspondence should be addressed to Yanjun Mao; maojunyan0626@163.com
}

Received 4 August 2021; Revised 26 October 2021; Accepted 28 October 2021; Published 13 November 2021

Academic Editor: Tao Huang

Copyright () 2021 Wenjie Xu et al. This is an open access article distributed under the Creative Commons Attribution License, which permits unrestricted use, distribution, and reproduction in any medium, provided the original work is properly cited.

\begin{abstract}
Background and Objective. Chronic obstructive pulmonary disease (COPD) features high morbidity. Different nursing interventions can relieve patient's burden. This investigation quantitatively assessed the impact of transitional care (TC) on self-management of patients with acute exacerbation of COPD based on a questionnaire survey. Methods. Clinical information of 78 COPD patients treated with TC (intervention group) or routine care (control group) in Shanghai Pulmonary Hospital during March 2019 and August 2020 was gathered. Patients were followed up for 3 months after discharge. The intervention group $(n=39)$ was subjected to a TC plan for 3 months to help patients and their family caregivers for self-management of COPD. TC was provided by specially trained nurses, and patients were supported by standardized tools. Nursing measures in the control group $(n=79)$ included transitional support for $30 \mathrm{~d}$ after hospital discharge. In this way, patients were guaranteed to follow discharge plans and transit to outpatient nursing. Patient's anxiety and depression symptoms, sleep quality, survival quality, mobility, and life quality at admission and after 3 months of discharge were assessed by Hospital Anxiety and Depression Scale, Pittsburgh Sleep Quality Index, Quality of Life Scale Abbreviated Version, Activity of Daily Life Scale, St. George's Respiratory Questionnaire, and COPD Assessment Test. Results. Except for anxiety and depression, patient's sleep quality, survival quality, mobility, and life quality in two groups were significantly improved. Moreover, average change of total CAT score during 3 months of intervention was -5.44 , while that in the control group was $-1.74(p=0.011)$. Improvement of survival quality of patients in the intervention group $(p=0.001)$ was markedly greater than that in the control group ( $p=0.016$ ). Conclusion. Altogether, TC based on quantification by questionnaire survey is beneficial to COPD patient's life quality and self-management.
\end{abstract}

\section{Introduction}

Chronic respiratory diseases $(\mathrm{CRD})$ are prime causes of global disability and death [1]. Chronic obstructive pulmonary disease (COPD) falls under the umbrella of CRD and is the most prevalent type. There were approximately 251 million COPD cases in the world in 2015, and this disease is expected to be the third factor of deaths in 2030 [2]. Common symptoms of COPD are shortness of breath, coughing, expectoration, or even eosinophilic airway inflammation [3]. Even if COPD can be preventable and treatable [4], resultant lower life quality and secondary psychological problems still exist including depression, anxiety, and dyspnea and impose heavy burden to patients, physically and mentally [5]. Earlier investigations suggested factors for pathogenesis of COPD, such as smoking, air pollution, infection, and vocation. Admission due to acute exacerbation of chronic obstructive pulmonary disease (AECOPD) is a grievous burden for individuals, like muscular dysfunction, dyspnea, and unable to move [6]. We devoted to developing effective nursing methods of releasing patient's negative emotions and increasing their life quality.

Transitional care (TC), also called continuous care or transitional nursing, is an extension of hospital care to 
guarantee the continuity of professional medical care for patients after discharge [7]. The purpose of TC is to increase patient's disease self-management, promote medical stability, and decline readmission rate via telephone follow-up, home visiting, and messages. TC has been proved to improve the prognosis of patients with chronic diseases [8, 9]. Moreover, TC can reduce medical costs by lowering emergency attendance rates and readmission rate [10]. An investigation indicated that nurses-led TC shows a positive impact on blood glucose control and treatment compliance, and lowers medical cost for diabetic patients after discharge [11]. A meta-analysis confirmed that TC significantly declines hospital readmission and mortality rate in COPD subjects [12]. For patients awaiting coronary artery bypass graft (CABG), TC can reduce anxiety, depression, and hospital times while enhancing cardiac self-efficacy and satisfaction with care [13].

In the above context, we conducted a retrospective analysis to quantitatively assess the impacts of TC on COPD patient's self-management based on a questionnaire survey. TC offers a rationale for the subsequent care of patients with COPD.

\section{Data and Methods}

2.1. Patient Inclusion. From March 2019 to August 2020, 79 COPD patients were treated in Shanghai Pulmonary Hospital Department of Respiratory and Critical Care Medicine No. 1 due to acute attack. They were diagnosed with COPD in accordance with Guideline for Diagnosis and Treatment of Chronic Obstructive Pulmonary Disease. All of the patients were older than 18. A patient who died during treatment was excluded, and 78 patients were finally included. The included patients were classified into the intervention group and control group (39 cases each). This study has been approved by the medical ethic committee of our hospital. Patient's information was used for research purpose only.

2.2. Intervention Group. Primary nurse was in charge of patient's care and evaluation from admission to follow-up and adopted one-to-one health education, including (I) respiratory muscle function exercise. (i) Pursed-lip breathing: the patient may be in the sitting, standing, or decubitus position. Patients are inhaling through their noses and exhaling through narrow mouths slowly with lips in the shape of a fish's mouth. The size of the labial contraction is adjusted by patient's own choice. The ratio of inhalation to exhalation is $1: 2$. The action is practiced twice with each time 5 min or as patient's abilities. (ii) Abdominal breathing: the patients take a comfortable position, and the whole body is relaxed. The abdomen is relaxed and bulged as inhaling, while it is contracted and sagged as exhaling. When exhaling, patients can press under ribs and on abdomen with their hands to promote abdominal muscles to contract and exhale. Abdominal breathing is practiced twice a day with each time $10 \mathrm{~min}$ or as their abilities. (iii) Respiratory exercise: collective respiratory rehabilitation exercises are carried out in the ward every morning. According to patient's situation, the exercise should be done step by step and acted according to their abilities. During hospitalization, patients are taught to master respiratory exercise. Atlas is distributed in convenience of their learning at home if they forget how to do it. (iv) Home oxygen therapy: patients are guided to take oxygen for no less than $15 \mathrm{~h}$ every day. It is advisable to take oxygen at night and before oxygen consumption is increased, such as meals and exercise. Oxygen uptake concentration is $1-2 \mathrm{~L} / \mathrm{min}$. (v) Nutrition guidance: it is advisable for patients to uptake foods with high protein, high fiber, moderate fat, moderate minerals and vitamins, low carbohydrate, low sugar, and low salt and drink more water, with frequent small meals. If significant hypoxia happens, oxygen therapy is recommended before or after a meal. (vi) Mental support: patiently listen to the patient and the difficulties they are encountering. Objectively analyze patient's state and treatment. Care, support, and encourage patients. Share successful cases with patients to build their confidence to overcome the disease. Guide watchers to encourage patients to do things according to their abilities in daily life. Increase patient's self-care ability and strengthen their role in daily life. Give guidance to watchers if they encounter difficulties during care. (vii) Telephone or WeChat follow-up was performed at the first and third months after discharge. Conduct care assessment and targeted health guidance for patients. The content includes signs and symptoms of COPD, complications or new symptoms, psychology, sleeping, home rehabilitation training, healthy behaviors, and environment. Aspect: (i) guide patients with correct and effective methods of coughing and hawking. Inform patients of self-monitoring symptoms and seeking medical advice in time if they feel uncomfortable. (ii) Evaluate whether patients participate in social activities and whether they are in a good mood. Encourage patients to actively participate in social activities. Timely psychological counseling shall be given to patients who are in an abnormal mood. (iii) Home rehabilitation training: evaluate whether patients exercise according to the discharge nursing prescription. Educate, coach, and follow up skills on the causes of poor compliance. Negotiate with patients to adjust nursing prescriptions. (iv) Healthy behaviors: evaluate whether patients take drugs, uptake oxygen, and do pursedlip breathing daily according to prescription. Assess their sleeping and diet conditions. Help patients establish a good lifestyle and correct healthy behaviors via health education and guidance. (v) Evaluate whether patients improve risk factors of home environment. If not, guide and supervise them to improve these factors. (vi) On-site examine the inhalation methods of various inhalers through respiratory chronic disease management nursing clinic, such as salmeterol xinafoate and fluticasone propionate powder for inhalation, tiotropium bromide powder for inhalation, and budesonide formoterol powder inhaler. Test patients who need to perform pursed-lip breathing, abdominal breathing, or breathing exercises. Evaluate patient's mastery and give correct guidance to increase their self-care ability.

2.3. Control Group. Patients in the control group only received routine care. COPD education curriculum was performed every two weeks for 1 month, including discharge 
TABLE 1: Baseline characteristics of patients in intervention and control groups.

\begin{tabular}{|c|c|c|c|c|}
\hline Characteristics & Intervention group $(n=39)$ & Control group $(n=39)$ & $t / \chi^{2} / Z$ & $p$ \\
\hline Age, years, median (IQR) & $66(62-68)$ & $64(59-68)$ & -1.514 & 0.130 \\
\hline \multicolumn{5}{|l|}{ Sex, $n(\%)$} \\
\hline Male & $36(92.3)$ & $34(87.2)$ & 0.557 & 0.711 \\
\hline Female & $3(7.7)$ & $5(12.8)$ & & \\
\hline BMI, kg/m², mean (SD) & $22.14 \pm 3.59$ & $21.24 \pm 3.80$ & 1.069 & 0.289 \\
\hline Disease course, month, median (IQR) & $72(36-120)$ & $64(21-178)$ & -0.510 & 0.610 \\
\hline Relevant family history, $n(\%)$ & $1(2.6)$ & $0(0.0)$ & 1.013 & 1.000 \\
\hline Drug allergy history, $n(\%)$ & $2(5.1)$ & $1(2.6)$ & 0.347 & 1.000 \\
\hline Care level, $n(\%)$ & & & 1.107 & 1.000 \\
\hline I & $1(2.6)$ & $0(0.0)$ & & \\
\hline II & $9(23.1)$ & $9(23.1)$ & & \\
\hline III & $27(69.2)$ & $28(71.8)$ & & \\
\hline IV & $2(5.1)$ & $2(5.1)$ & & \\
\hline Family average annual income $\leq 50000 \mathrm{CNY}, n(\%)$ & $13(34.2)$ & $15(38.5)$ & 0.150 & 0.814 \\
\hline Profession, $n(\%)$ & & & 0.217 & 0.816 \\
\hline Retire & $23(59.0)$ & $25(64.1)$ & & \\
\hline Others & $16(41.0)$ & $14(35.9)$ & & \\
\hline Current smoking, $n(\%)$ & $25(64.1)$ & $23(59.0)$ & 0.217 & 0.816 \\
\hline Payment of medical expense, $n(\%)$ & & & 0.963 & 0.462 \\
\hline Medical insurance & $25(64.1)$ & $29(74.4)$ & & \\
\hline Self-expense/partial expense & $14(35.9)$ & $10(25.6)$ & & \\
\hline Education degree, $n(\%)$ & & & 0.396 & 0.908 \\
\hline Primary school & $12(30.8)$ & $11(28.2)$ & & \\
\hline Junior high school & $21(53.8)$ & $20(51.3)$ & & \\
\hline Senior high school & $6(15.4)$ & $8(20.5)$ & & \\
\hline \multicolumn{5}{|l|}{ Marriage status, $n(\%)$} \\
\hline Married & $39(100.00)$ & $39(100.00)$ & & \\
\hline Single & $0(0.0)$ & $0(0.0)$ & & \\
\hline Breathing function exercise history, $n(\%)$ & $0(0.0)$ & $1(2.6)$ & 1.013 & 1.000 \\
\hline Complications and drugs, $n(\%)$ & $3(7.7)$ & $2(5.1)$ & 0.214 & 1.000 \\
\hline SpO2, median (IQR) & $96(95-97)$ & $96(95-97)$ & -0.068 & 0.946 \\
\hline Hospital day, $d$, median (IQR) & $6(6-8)$ & $7(6-8)$ & -0.475 & 0.635 \\
\hline
\end{tabular}

SpO2: oxyhemoglobin saturation.

process guidance, disease factor information, pulmonary rehabilitation, and home oxygen therapy.

2.4. Evaluation Index. Patient's anxiety and depression symptoms, sleep quality, survival quality, mobility, and life quality at admission and after 3 months of discharge were assessed by Hospital Anxiety and Depression Scale (HADS), Pittsburgh Sleep Quality Index (PSQI), Quality of Life Scale Abbreviated Version (QOL-BREF), Activity of Daily Life Scale (Barthel index), St. George's Respiratory Questionnaire (SGRQ), and COPD Assessment Test (CAT) [14]. Details were provided in the Supplementary material.

HADS [15] was employed to assess the anxiety and depression of the subjects in this investigation. This scale contains 7 HADS-anxiety items and 7 HADS-depression items. Each item was equipped with a score from 0 to 3 . A lower score presents more serious anxiety or depression.
Patients with total score $\leq 8$ was in a state of anxiety or depression.

PSQI [16] was used to assess the sleep quality of subjects during the latest month. This scale consists of 19 selfassessment and 5 peer assessment items, among which the $19^{\text {th }}$ self-assessment and $5^{\text {th }}$ peer assessment items are not involved in scoring. Here, only the 18 self-assessment items involved in scoring were introduced (see Supplementary material). The 18 items constitute 7 parts, and each part was scored according to 0-3. The cumulative scores of each part were PSQI scores within 0-21. Higher score presents poor sleep quality.

QOL-BREF [17] is a self-management questionnaire with 26 items scored on a 5-point scale. Areas of assessment include physiological field, psychological field, social relationship field, environmental field, subjective feeling of quality of life, subjective feeling of health status, family friction, 
TABLE 2: Scoring results of patients in the intervention and control groups.

\begin{tabular}{|c|c|c|c|c|c|c|}
\hline & \multicolumn{3}{|c|}{ Intervention group $(n=39)$} & \multicolumn{3}{|c|}{ Control group $(n=39)$} \\
\hline & $\mathrm{T} 1$ & $\mathrm{~T} 2$ & $\triangle \mathrm{T}$ & T1 & $\mathrm{T} 2$ & $\triangle \mathrm{T}$ \\
\hline HADS & $36.03 \pm 4.96$ & $36.85 \pm 2.85$ & $0.82 \pm 5.34$ & $35.87 \pm 5.55$ & $35.79 \pm 4.03$ & $-0.08 \pm 4.02$ \\
\hline PSQI & $11.95 \pm 9.35$ & $7.87 \pm 6.14^{*}$ & $-4.08 \pm 7.33$ & $12.23 \pm 8.97$ & $10.79 \pm 7.83^{*}$ & $-1.44 \pm 3.66$ \\
\hline QOL-BREF & $54.78 \pm 8.69$ & $59.74 \pm 7.77^{*}$ & $4.96 \pm 8.95$ & $54.33 \pm 7.92$ & $57.03 \pm 8.15^{*}$ & $2.70 \pm 6.65$ \\
\hline $\begin{array}{l}\text { Activity of daily life scale } \\
\text { (Barthel index) }\end{array}$ & $76.03 \pm 14.79$ & $85.64 \pm 16.23^{*}$ & $9.62 \pm 23.41$ & $76.28 \pm 13.29$ & $79.82 \pm 11.66^{*}$ & $3.54 \pm 8.86$ \\
\hline SGRQ & $51.70 \pm 19.41$ & $39.45 \pm 23.44^{*}$ & $-12.25 \pm 24.98$ & $52.87 \pm 16.20$ & $47.90 \pm 15.00^{*}$ & $-4.97 \pm 11.27$ \\
\hline CAT & $19.85 \pm 5.37$ & $14.41 \pm 7.51^{*}$ & $-5.44 \pm 7.04$ & $20.18 \pm 5.07$ & $18.44 \pm 5.92^{*}$ & $-1.74 \pm 5.29^{\boldsymbol{\Lambda}}$ \\
\hline
\end{tabular}

T1: on admission; T2: postdischarge third month; $\triangle \mathrm{T}$ : pre-post changes; ${ }^{*} p<0.05$ compared with before admission in each group, respectively. ${ }^{\mathbf{A}} p<0.05$ compared with changes in the control group.

TABLE 3: $p$ values of two groups after scale scoring.

\begin{tabular}{lccc}
\hline & $\begin{array}{c}\text { Intervention group }(n=39) \\
p \text { values before and after } \\
\text { treatment }\end{array}$ & $\begin{array}{c}\text { Control group }(n=39) \\
p \text { values before and after } \\
\text { treatment }\end{array}$ & $\begin{array}{c}p \text { value (differences between groups after } \\
\text { treatment) }\end{array}$ \\
\hline HADS & 0.343 & 0.906 & 0.404 \\
PSQI & 0.001 & 0.019 & 0.048 \\
QOL-BREF & 0.001 & 0.016 & 0.210 \\
Activity of daily life scale (Barthel & 0.014 & 0.017 & 0.134 \\
index) & 0.004 & 0.009 & 0.101 \\
SGRQ & $<0.001$ & 0.046 & 0.011 \\
CAT & &
\end{tabular}

and appetite. Scores range from 0 to 100 . Higher score refers to higher quality of life. Primitive domain scores should be transformed to 0-100 level in convenience of comparison with other datasets. In this transformation, the possible lowest score was set to 0 while the possible highest score was set to 100 . Scores among values refer to percentage of total possible scores achieved.

Activity of Daily life Scale (Barthel index) [18] includes 10 items. According to the needs for help and levels of help, the scores were divided into four functional levels $(0,5,10$, 15). The total score is 100 . Higher score denotes stronger independence and little dependence.

Health-related life quality was assessed by SGRQ [19], including symptoms, activity, and influence. Total score was considered to be the sum of each domain score. Higher score refers to poor life quality.

The impact of COPD on health status is assessed by CAT [20]. CAT evaluated symptoms like cough, hawking, and dyspnea and the impact of disease on daily life. CAT consists of 8 items, with each item scored within $0-5(0=$ no deficiency). Total score is within 0-40. 0-10, 11-20, 2130, and 31-40 refer to light, mild, severe, and extremely severe clinical impacts. Lower CAT score denotes higher life quality.

2.5. Statistical Analysis. Data were processed by SPS 25.0 software. The measurement data were first tested for normality. Data that fit normality were displayed as mean value \pm standard deviation. Independent sample $t$ test was adopted for comparison between two groups. Data that did not fit normality were displayed as IQR. Wilcoxon rank sum test was adopted for comparison among groups. Two category data were denoted as numbers and percentages, and comparison between two groups was chi-square or Fisher exact test. Paired sample $t$-test was used for each score between two groups, scores at admission and after 3 months of discharge in each group. Score change in each group was analyzed by independent sample $t$ test. $p<0.05$ denoted statistical significance.

\section{Results}

3.1. Patient's Baseline Characteristics. As shown in Table 1, no significant differences were found with respect to age, sex, body mass index (BMI), disease course, relevant family history, drug allergy history, care level, family average annual income, smoking, profession, payment of medical expense, education degree, marriage status, breathing function exercise history, complications, and medicine of patients in two groups. The two groups were comparable.

3.2. Scoring Results. There were no marked differences in anxiety and depression, sleep status, survival quality, Barthel index, SGRQ, and CAT of patients in two groups. After 3 months of discharge, each score was remarkably improved except for anxiety and depression. Moreover, independent 
sample $t$-test revealed markedly higher improvement of survival quality of patients in the intervention group than that in the control group. As shown in Tables 2 and 3, TC based on a questionnaire survey could conspicuously enhance COPD patient's life quality.

\section{Discussion}

This investigation quantitatively compared the impacts of $\mathrm{TC}$ and routine care on disease self-management of COPD patients based on a questionnaire survey. The improvement of survival quality of patients with PT was more significant than patients with routine care. Nevertheless, their anxiety and depression, sleep quality, survival quality, and mobility were not significantly different.

A randomized clinical trial showed that the combination of TC and long-term self-management support lead to more COPD-relevant hospitalizations and emergency visits, without life quality improvement [21]. This study also mentioned that acute care events occur more often in COPD patients who are in a highly activated status when admitted to the hospital. This might trigger inconsistency between its results and ours. In contrast, another study indicated that a website-based TC plan can enhance the life quality and glycemic control of patients with type 2 diabetes after discharge [11]. Likewise, a randomized controlled trial assessed nurseled TC for old people with open heart surgery. It was suggested that patient's life quality and functional autonomy are markedly increased, while rehospitalization and hospital readmission rates after discharge are conspicuously decreased [22]. TC also improves acute attack patient's life quality [23]. TC conspicuously decreases risk for admission relevant to COPD and caused by COPD [12]. These hold an agreement with our investigation, suggestive of superiority of TC on improving COPD patient's life quality.

Earlier studies presented prevalence of COPD among males than females; therefore, we researched more males. However, with increased smoking of women in highincome countries and more risks for exposure to indoor air pollution (such as biomass fuels for cooking and heating) of women in low-income countries, the future morbidity of COPD among men and women may be equal [24, 25]. Incremental study proved that female is a major risk factor for depression in adult patients with COPD [26]. Of note, intervention time, caregiver type, and telephone visit may affect the outcomes of TC. For example, TC based on app increases self-efficacy of spinal cord injury patients, while it does not improve quality of life significantly [27]. In this investigation, we evaluated COPD patient's life quality via various questionnaires and found positive enhancement. Most previous studies evaluated patient's readmission rates rather than life quality based on various scales. Our investigation presents reliable results in support of the improvement of TC on COPD patients.

However, this investigation has several limitations. First of all, this investigation only included 3-month outcomes of TC based on a questionnaire survey. Thus, the longterm effect, such as readmission rate, was not obtained accurately. Moreover, patient's compliance with drugs should be considered. Secondly, we only collected samples from a hospital, not representative of overall conditions of patients in other regions. COPD morbidity is different in varying areas [28]. Lastly, the sample size in this investigation is small; thus, patient's characteristics themselves may influence the results.

Overall, this investigation confirmed that TC based on a questionnaire survey might improve life quality of COPD patients and perform positive effects on disease selfmanagement. It is necessary to verify the results with more samples and perfect TC programs.

\section{Data Availability}

The data used to support the findings of this study are included within the article.

\section{Consent}

All authors consent to submit the manuscript for publication.

\section{Conflicts of Interest}

The authors declare that they have no potential conflicts of interest.

\section{Authors' Contributions}

WJ, $\mathrm{HH}$, and $\mathrm{YJ}$ contributed to data analysis, drafting and revising the article, gave final approval of the version to be published, and agreed to be accountable for all aspects of the work.

\section{Acknowledgments}

This study was supported by the funds from Research on the Construction and Application of COPD Continuing Nursing Program Based on Pulmonary Specialty Alliance (201840107).

\section{Supplementary Materials}

I. Hospital Anxiety and Depression Scale (HADS) (Supplementary Materials)

\section{References}

[1] GBD Chronic Respiratory Disease Collaborators, "Prevalence and attributable health burden of chronic respiratory diseases, 1990-2017: a systematic analysis for the Global Burden of Disease Study 2017," The Lancet Respiratory Medicine, vol. 8, no. 6, pp. 585-596, 2020.

[2] F. Herse, T. Kiljander, and L. Lehtimaki, "Annual costs of chronic obstructive pulmonary disease in Finland during 1996-2006 and a prediction model for 2007-2030," npj Primary Care Respiratory Medicine, vol. 25, no. 1, article 15015, 2015.

[3] D. Singh, A. Agusti, A. Anzueto et al., "Global strategy for the diagnosis, management, and prevention of chronic obstructive 
lung disease: the GOLD science committee report 2019," The European Respiratory Journal, vol. 53, no. 5, p. 1900164, 2019.

[4] K. F. Rabe and H. Watz, "Chronic obstructive pulmonary disease," Lancet, vol. 389, no. 10082, pp. 1931-1940, 2017.

[5] A. Tselebis, A. Pachi, I. Ilias et al., "Strategies to improve anxiety and depression in patients with COPD: a mental health perspective," Neuropsychiatric Disease and Treatment, vol. 12, pp. 297-328, 2016.

[6] K. Vermeersch, M. Gabrovska, J. Aumann et al., "Azithromycin during acute chronic obstructive pulmonary disease exacerbations requiring hospitalization (BACE). A multicenter, randomized, double-blind, placebo-controlled trial," American Journal of Respiratory and Critical Care Medicine, vol. 200, no. 7, pp. 857-868, 2019.

[7] H. Shahsavari, M. Zarei, and J. Aliheydari Mamaghani, "Transitional care: concept analysis using Rodgers' evolutionary approach," International Journal of Nursing Studies, vol. 99, article 103387, 2019.

[8] Q. Tu, L. D. Xiao, S. Ullah, J. Fuller, and H. Du, "A transitional care intervention for hypertension control for older people with diabetes: a cluster randomized controlled trial," Journal of Advanced Nursing, vol. 76, no. 10, pp. 2696-2708, 2020.

[9] Y. H. Bae-Shaaw, H. Eom, R. F. Chun, and D. Steven Fox, "Real-world evidence on impact of a pharmacist-led transitional care program on 30- and 90-day readmissions after acute care episodes," American Journal of Health-System Pharmacy, vol. 77, no. 7, pp. 535-545, 2020.

[10] M. Schapira, M. B. Outumuro, F. Giber et al., "Geriatric comanagement and interdisciplinary transitional care reduced hospital readmissions in frail older patients in Argentina: results from a randomized controlled trial," Aging Clinical and Experimental Research, 2021.

[11] Q. Y. Lyu, J. W. Huang, Y. X. Li et al., "Effects of a nurse led web-based transitional care program on the glycemic control and quality of life post hospital discharge in patients with type 2 diabetes: a randomized controlled trial," International Journal of Nursing Studies, vol. 119, article 103929, 2021.

[12] E. S. Ridwan, H. Hadi, Y. L. Wu, and P. S. Tsai, "Effects of transitional care on hospital readmission and mortality rate in subjects with COPD: a systematic review and meta-analysis," Respiratory Care, vol. 64, no. 9, pp. 1146-1156, 2019.

[13] A. Yuroong, U. Asdornwised, W. Pinyopasakul, W. Wongkornrat, and N. Chansatitporn, "The effectiveness of the transitional care program among people awaiting coronary artery bypass graft surgery: a randomized control trial," Journal of Nursing Scholarship, vol. 53, no. 5, pp. 585-594, 2021.

[14] A. Shah, N. Ayas, W. C. Tan et al., "Sleep quality and nocturnal symptoms in a community-based COPD cohort," COPD, vol. 17, no. 1, pp. 40-48, 2020.

[15] A. S. Zigmond and R. P. Snaith, "The hospital anxiety and depression scale," Acta Psychiatrica Scandinavica, vol. 67, no. 6, pp. 361-370, 1983.

[16] L. Nobeschi, J. Zangirolami-Raimundo, P. K. Cordoni et al., "Evaluation of sleep quality and daytime somnolence in patients with chronic obstructive pulmonary disease in pulmonary rehabilitation," BMC Pulmonary Medicine, vol. 20, no. 1, p. 14, 2020.

[17] The WHOQOL Group, "The World Health Organization Quality of Life assessment (WHOQOL): position paper from the World Health Organization," Social Science \& Medicine, vol. 41, no. 10, pp. 1403-1409, 1995.

[18] M. Vitacca, A. Malovini, B. Balbi et al., "Minimal clinically important difference in Barthel index dyspnea in patients with COPD," International Journal of Chronic Obstructive Pulmonary Disease, vol. Volume 15, pp. 2591-2599, 2020.

[19] P. W. Jones and S. G.'s. Respiratory, "St. George's Respiratory Questionnaire: MCID," COPD, vol. 2, no. 1, pp. 75-79, 2005.

[20] P. W. Jones, G. Harding, P. Berry, I. Wiklund, W. H. Chen, and N. Kline Leidy, "Development and first validation of the COPD assessment test," The European Respiratory Journal, vol. 34, no. 3, pp. 648-654, 2009.

[21] H. Aboumatar, M. Naqibuddin, S. Chung et al., "Effect of a hospital-initiated program combining transitional care and long-term self-management support on outcomes of patients hospitalized with chronic obstructive pulmonary disease: a randomized clinical trial," JAMA, vol. 322 , no. 14, pp. 13711380, 2019.

[22] S. Coskun and S. Duygulu, "The effects of nurse led transitional care model on elderly patients undergoing open heart surgery: a randomized controlled trial," European Journal of Cardiovascular Nursing, 2021.

[23] T. O. Oyesanya, C. Loflin, L. Byom et al., "Transitions of care interventions to improve quality of life among patients hospitalized with acute conditions: a systematic literature review," Health and Quality of Life Outcomes, vol. 19, no. 1, p. 36, 2021.

[24] K. K. Lee, R. Bing, J. Kiang et al., "Adverse health effects associated with household air pollution: a systematic review, metaanalysis, and burden estimation study," The Lancet Global Health, vol. 8, no. 11, pp. e1427-e1434, 2020.

[25] J. R. Balmes, "Household air pollution from domestic combustion of solid fuels and health," The Journal of Allergy and Clinical Immunology, vol. 143, no. 6, pp. 1979-1987, 2019.

[26] J. S. Choi, S. H. Kwak, N. H. Son, J. W. Oh, S. Lee, and E. H. Lee, "Sex differences in risk factors for depressive symptoms in patients with COPD: the 2014 and 2016 Korea National Health and Nutrition Examination Survey," BMC Pulmonary Medicine, vol. 21, no. 1, p. 180, 2021.

[27] T. Liu, S. Xie, Y. Wang et al., "Effects of app-based transitional care on the self-efficacy and quality of life of patients with spinal cord injury in China: randomized controlled trial," JMIR mHealth and uHealth, vol. 9, no. 4, article e22960, 2021.

[28] D. Adeloye, S. Chua, C. Lee et al., "Global and regional estimates of COPD prevalence: systematic review and meta-analysis," Journal of Global Health, vol. 5, no. 2, article 020415, 2015. 